\title{
Lung cancer: new tools for surgery
}

\author{
Sanjoy Roy*,1 \\ ${ }^{1}$ Ethicon/Johnson \& Johnson Medical Devices, Cincinnati, OH, USA \\ *Author for correspondence: sroy13@its.jnj.com
}

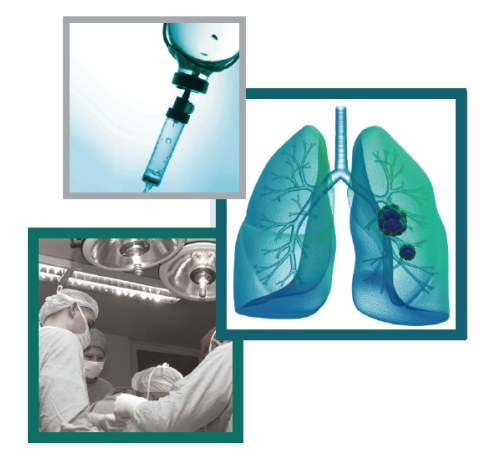

Sanjoy Roy speaks to Alfie Gleeson, Commissioning Editor: Sanjoy Roy is the Director of Franchise Health Economics and Market Access at Ethicon, Inc. - part of the Johnson and Johnson Medical Device Companies (OH, USA). He has a Bachelor's degree in Pharmacy from Jadavpur University (West Bengal, India), a Master's degree in Health Outcomes and Policy from West Virginia University (WV, USA) and a Professional Certificate in Strategic Marketing from Harvard University (MA, USA). He is a health economist and outcomes researcher with over two decades of work experience in the pharmaceutical and medical devices industry - both in commercial and in research functions. Ethicon has been making significant contributions to surgery, particularly in the suture field, for over 60 years. From the first sutures to the development of minimally invasive procedures they have revolutionized surgery more than once. Sanjoy Roy talks to Lung Cancer Management about how Ethicon has shaped surgery, and how new tools that Ethicon has developed have improved lung cancer surgery outcomes and reduced the economic burden of lung cancer treatment. Finally, we look at the future of lung cancer surgery and how it may change in the light of new technologies and the global burden of disease and healthcare costs.

First draft submitted: 30 July 2018; Accepted for publication: 22 August 2018; Published online:

1 November 2018

\section{How has Ethicon shaped surgery?}

Ethicon (OH, USA) has been at the forefront of surgical innovation for many decades now. Ethicon has a long history of providing innovative surgical devices to enable optimal surgical care for lung cancer patients. Our portfolio of sutures, hemostatic agents, staplers and energy devices have been designed based on the fundamental science of device-tissue interaction and an understanding of the specific requirements of each surgical job and tissue type. With the increasing digitalization of healthcare, we are now able to validate the impact of these innovations on surgical outcomes and thereby complete the innovation cycle.

Furthermore, Ethicon offers unsurpassed professional education and training to help build strong surgical proficiency for lung cancer surgery globally.

Overall, Ethicon's mission is to shape the future of surgery. A significant component of that is working with surgeons and other care providers to reduce surgical complications and optimize lung cancer surgery for patients while ensuring economic benefits for the healthcare system.

\section{What new tools and/or procedures that Ethicon have produced are you excited about?}

Ethicon's broad surgical product portfolio offers many devices that enable surgeons to deliver optimal surgical treatment for lung cancer. Among the latest innovations from Ethicon are two endoscopic staplers that together offer a best-in-class option for the most critical transection jobs during lung cancer surgery.

The ECHELON FLEX ${ }^{T M}$ Powered Vascular Stapler is an example of a device Ethicon has designed specifically for the most critical step in any anatomical lung resection: transecting pulmonary vessels. It is a tissue-specific stapler that enables improved access and more precise placement on fragile pulmonary vessels because of its curved tip, narrow anvil, wide range of articulation and small shaft diameter.

Another innovative device in our portfolio is the ECHELON FLEX GST System. We know that lung parenchyma thickness is highly variable, depending on the region of the lung on which the surgeon is working. This endoscopic stapler enables a thoracic surgeon to transect as intended on the bronchus and parenchyma, providing exceptional staple line integrity on the broadest range of thoracic tissue thickness.

Together, these surgical devices enable surgeons to perform transections with the level of precision they desire, thus reducing variability of outcomes.

Future $\because$ Medicine 


\section{What implications do these tools/procedures have for lung cancer treatment?}

A retrospective real-world database analysis that was recently published [1] in the peer-reviewed journal, Advances in Therapy, demonstrated that when ECHELON FLEX Powered Vascular Stapler and another ETHICON Powered Stapler were used together in video-assisted thoracoscopic surgery lobectomy procedures, these devices were associated with improved clinical outcomes for patients and significant economic benefits for hospitals compared with competitive device options. Procedures, where these two staplers were used together, were associated with a $66 \%$ reduction in hemostasis-related complications and a reduction in length of stay by almost a full day - without increasing costs.

As such, patients with lung cancer who are eligible for surgical treatment can potentially benefit from lower complications with the use of these devices for the most critical steps in their procedure - hopefully leading to improved longer term surgical and oncologic outcomes, as suggested in the clinical literature.

\section{How can innovations in surgery improve the economic burden of cancer?}

The global economic burden of disease continues to increase, and with aging populations and slowing economic growth worldwide, cost of healthcare is only expected to get even more challenging in the future. As a healthcare and medical device innovator, Ethicon/Johnson \& Johnson, (OH, USA) considers it to be our responsibility to partner with healthcare providers to develop innovations that help to bend this cost curve.

Surgery has been established as an important part of the treatment strategy for early-stage lung cancer patients. Surgical complications could adversely impact follow-up cancer care and long-term patient oncologic outcomes and survival, as well as, significantly increase the cost of care and lost productivity. As such, reducing the risk of complications is of paramount importance in lung cancer surgery. Device innovations that are associated with reduced complications, therefore, can potentially offer significant improvement to surgical, oncologic and economic outcomes of lung cancer.

The driving force behind innovation for lung cancer treatment at Ethicon is the desire to reduce surgical complications without increasing total costs of the procedure. An example of such device innovation was demonstrated in a recently published paper [1], where the use of Ethicon's new powered and thoracic tissue-specific endoscopic staplers was associated with an estimated $9 \%$ reduction of total hospital costs - primarily driven by an associated decrease in hemostasis-related complications and almost a day shorter hospital stay.

\section{What impact will technologies such as robotics \& artificial intelligence have on lung cancer surgery in the next 5 years?}

Robotics, along with laparoscopic technologies, are enabling access to minimally invasive surgeries for more lung cancer patients - with improved outcomes and less trauma compared with open surgeries. With further enhancement of surgical robotic technologies, and availability of AI-driven visualization and training, lung cancer surgeries are expected to continue to be further standardized and potentially could become an optimal option for cancer treatment for more patients worldwide - with improved outcomes, cost-effectively.

\section{What future projects are Ethicon working on to innovate lung cancer treatment?}

Ethicon, and, more broadly, Johnson \& Johnson, is committed to bringing innovation to market which addresses the significant unmet needs remaining in the treatment of lung cancer patients. Ethicon specifically focuses our innovation efforts on surgical oncology, but because Johnson \& Johnson is broadly based across healthcare we are able to develop new innovation across a spectrum of technology and intervention settings. An example of our broader investments in innovation is the recently announced 5-year alliance with Boston University aimed at fundamentally changing the way lung cancer is detected and treated.

What advice would you give young scientists, researchers \& students who would like a career in health economics, especially given the current climate of scientific mistrust \& uncertainty?

For anyone considering a career in health economics, my advice would be to stay curious and to stay reliable.

With an almost simultaneous explosion of the global burden of disease and the costs of healthcare, it is up to the health economists of tomorrow to make sure that patients can continue to have access to and benefit from meaningful, cost-effective innovation that saves human lives and enhances human productivity around the world.

Their curiosity will allow tomorrow's health economic researchers to go beyond the boundaries of traditional sources of evidence of value of health technologies, and the oncoming deluge of data would need them to be careful 
and creative in using those to develop reliable, meaningful and actionable insights that inform decisions about access to care.

It is already great to be in or to be considering a career in health economics today, and the future holds even more exciting opportunities to impact human health in ever more meaningful ways.

\section{Disclaimer}

The opinions expressed in this interview are those of the interviewee and do not necessarily reflect the views of Future Medicine Ltd.

\section{Financial \& competing interests disclosure}

$\mathrm{S}$ Roy is an employee and shareholder of Johnson and Johnson. The research featured in the interview was funded by Ethicon/Johnson and Johnson, who manufacture surgical devices included in the analysis. The authors have no other relevant affiliations or financialinvolvement with any organization or entity with a financial interest in or financial conflict with the subject matter or materials discussed in the manuscript apart from those disclosed.

No writing assistance was utilized in the production of this manuscript.

\section{Open access}

This work is licensed under the Attribution-NonCommercial-NoDerivatives 4.0 Unported License. To view a copy of this license, visit http://creativecommons.org/licenses/by-nc-nd/4.0/

\section{References}

1. Miller DL, Roy S, Kassis ES, Yadalam S, Ramisetti S, Johnston SS. Impact of powered and tissue-specific endoscopic stapling technology on clinical and economic outcomes of video-assisted thoracic surgery lobectomy procedures: a retrospective, observational study. $A d v$. Ther. 35, 707 (2018) doi: doi.org/10.1007/s12325-018-0679-z. 
